\title{
Preoperative Serum Markers Prognostic Evaluation in Colon Cancer Patients
}

\author{
Luigina Graziosi*, Marino Elisabetta, Alberto Rebonato and Annibale Donini
}

Department of Surgical Sciences, Radiologichee Odontostomatologiche, University of Perugia, Italy

\begin{abstract}
Aim: Demonstrate that preoperative tumor markers are prognostic factor in colon rectal cancer and their high levels are correlated with bad prognosis.

Methods: We retrospectively analyzed two hundred and thirty-one patients affected by colorectal cancer who underwent radical surgery between January 2012 and August 2016 from a prospectively collected database. The study group consisted of 224 patients: 132 men and 92 women. Demographic details, surgical procedure, histopathologic diagnosis, and survival data were prospectively collected and retrospectively reviewed for this study. Normal cut off values for Carcinoembryonic antigen (CEA) and Carbohydrate Antigen 19.9 (CA 19.9) were respectively $5 \mathrm{ng} / \mathrm{ml}$ and $35 \mathrm{Ul} / \mathrm{ml}$. A P-value $<0.05$ was deemed to be statistically significant.

Results: Tumor location was 94 times on ascending colon (42\%), 13 on transverse colon $(5.8 \%), 77$ on descending colon $(34.4 \%)$ and 40 on rectum (17.8\%). All tumors were histologically diagnosed as adenocarcinoma of the colon-rectum and staged according to the TNM staging system. Preoperative serum CEA level was significantly associated only with $\mathrm{T}$ stage and serum albumin level; whereas there were no statistically significant differences between preoperative serum CA19.9 and patients' clinical pathologic characteristics. Overall survival (OS) and disease-free survival (DFS) showed a statistically significant difference in the two groups of patients who are dichotomized according to the normal cut-off value of CEA and $\mathrm{Ca}$ 19.9. At the multivariate analysis both preoperative CEA and Ca 19.9 resulted as independent prognostic factor for survival with a $p<0.05$.
\end{abstract}

Conclusion: These two tumor markers could have a role as prognostic factors leading to a stricter postsurgical follow up in those patients with elevated preoperative values.

Core tip: Preoprative measurement of Cea and Ca19.9 is a cheap and routine exam. Their role could help to identify patients with poor prognosis in the preoperative period and to strictly follow up them in the post-surgical setting.

Keywords: Colon cancer; Tumoral markers; Radical surgery; Predictive value

\section{Introduction}

Colorectal cancer (CRC) accounts for $13 \%$ of all cancers, it represents the third most common neoplasia and it stands for the second leading cause of cancer death in the 27 countries of the European Union $[1,2]$. Prognosis of CRC patients is mainly dependent on several factors: pathological, clinical and biological. Although pathologic stage is useful and essential for predicting prognosis in CRC patients, it is difficult to determine in an accurate way the stage prior to the surgical treatment. It is also well known that patients with the same pathological stage show survival heterogeneity. Thus, it is necessary to identify promising prognostic factors that could preoperatively identify patients at high risk of recurrences after surgery or with a bad survival prognosis. Carcinoembrionic Antigen (CEA) and Carbohydrate 19.9 (CA 19.9) are the most common used tumor-associated antigens used in the pre- and post-operative management of patients affected by colon cancer. They can be inexpensively and easily measured increasing their applicability in all patients who will undergo colorectal surgery. There are several reports in the literature demonstrating their useful correlation to post-surgical [3-5] recurrence but there are few and controversial studies that evaluate their pre-operative prognostic values in both overall and disease-free survival. Our aim is to analyze the survival significance of preoperative serum CEA and CA 19.9 values in patients undergoing potential macroscopic curative surgical procedure for CRC.

\section{Patients and Methods}

\section{Patients}

We retrospectively analyzed data on two hundred and thirty-one patients with colorectal cancer who underwent macroscopically curative surgery between January 2012 and August 2016.
Radical and curative surgery was defined by the absence of any gross residual tumors from the surgical bed and by a negative surgical resection margin. Patients without pre-operative measurements of the serum levels of tumor markers or without a radical surgery were excluded from the study. The study group consisted of 224 patients with 132 men and 92 women. Average age was 72.5 (s.d. \pm 11.4 yo) years old. The pathological stage was based on the $7^{\text {th }}$ edition of American Join Committee on Cancer, Colon and Rectum cancer staging. In our series 48 patients were stage I $(21.4 \%), 82(36.6 \%)$, were stage II, $62(27.7 \%)$ were stage III, and $32(14.3 \%)$ were stage IV.

\section{Data collection}

Demographic details, surgical procedure, histopathologic diagnosis, and survival data were prospectively collected and retrospectively reviewed for this study. Normal cut off values for CEA and CA 19.9 were respectively $5 \mathrm{ng} / \mathrm{ml}$ and $35 \mathrm{UI} / \mathrm{ml}$. Disease free survival (DFS) was defined as the time from surgery to the first recurrence of the disease.

*Corresponding author: Luigina Graziosi, PHD, MD, Department of Surgical Sciences, Radiologiche e Odontostomatologiche, Nuova Facoltà di Medicina e Chirurgia Sant'Andrea delle Fratte, 06132 Perugia, Italy, Tel: 075/5786445; Fax: 075/5786445; Email: luiginagraziosi@yahoo.it

Received January 24, 2018; Accepted February 15, 2018; Published February 19, 2018

Citation: Graziosi L, Elisabetta M, Rebonato A, Donini A (2018) Preoperative Serum Markers Prognostic Evaluation in Colon Cancer Patients. J Cancer Sci Ther 10: 022-026. doi:10.4172/1948-5956.1000511

Copyright: @ 2018 Graziosi L, et al. This is an open-access article distributed under the terms of the Creative Commons Attribution License, which permits unrestricted use, distribution, and reproduction in any medium, provided the original author and source are credited. 
Citation: Graziosi L, Elisabetta M, Rebonato A, Donini A (2018) Preoperative Serum Markers Prognostic Evaluation in Colon Cancer Patients. J Cancer Sci Ther 10: 022-026. doi:10.4172/1948-5956.1000511

Overall Survival (OS) was defined as the time either from surgery or from the last follow-up to death. All the studied patients were dichotomized at the normal value of CEA and Ca 19.9.

\section{Blood sample analyses}

Venous blood sampling was taken before surgery and collected in ethylene diamine tetra acetic acid (EDTA) containing tube. CEA and Ca 19.9 were detected by ELISA technique.

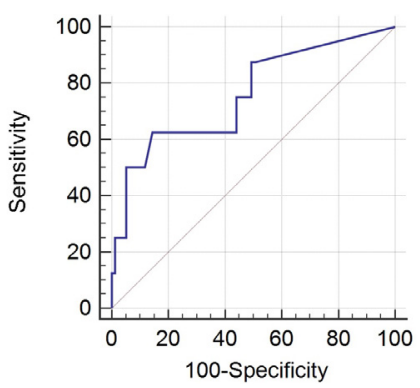

Figure 1: CEA sieric value (cutoff $5 \mathrm{ng} / \mathrm{ml}$ ) and 2Y-OS Roc CURVE; Sensitivity $62.5 \%$, Specificity $85.7 \%$.

\begin{tabular}{|c|c|c|c|}
\hline Variables & Low CEA & High CEA & $\mathbf{P}$ \\
\hline \multicolumn{4}{|c|}{ Age } \\
\hline$<65$ & 38 & 10 & \multirow{2}{*}{0.08} \\
\hline$>65$ & 109 & 60 & \\
\hline \multicolumn{4}{|c|}{ Gender } \\
\hline Female & 59 & 29 & \multirow{2}{*}{0.9} \\
\hline Male & 88 & 41 & \\
\hline \multicolumn{4}{|c|}{ Tumour Site } \\
\hline Right & 59 & 31 & \multirow{4}{*}{0.2} \\
\hline Left & 48 & 27 & \\
\hline Rectum & 28 & 11 & \\
\hline Transverse & 12 & 1 & \\
\hline \multicolumn{4}{|c|}{ T stage } \\
\hline 1 & 14 & 0 & \multirow{4}{*}{0.003} \\
\hline 2 & 37 & 10 & \\
\hline 3 & 84 & 48 & \\
\hline 4 & 12 & 12 & \\
\hline \multicolumn{4}{|c|}{ N stage } \\
\hline 0 & 95 & 36 & \multirow{4}{*}{0.2} \\
\hline 1 & 30 & 18 & \\
\hline 2 & 21 & 16 & \\
\hline 3 & 1 & 0 & \\
\hline \multicolumn{4}{|c|}{ N stage } \\
\hline Positive & 52 & 34 & \multirow{2}{*}{0.08} \\
\hline Negative & 95 & 36 & \\
\hline \multicolumn{4}{|c|}{ Lymph. Harvasted } \\
\hline$<12$ & 43 & 14 & \multirow{2}{*}{0.1} \\
\hline$>12$ & 104 & 56 & \\
\hline \multicolumn{4}{|c|}{ Sieric Albumin } \\
\hline$<3.5$ & 32 & 25 & \multirow{2}{*}{0.04} \\
\hline$>3.5$ & 103 & 40 & \\
\hline \multicolumn{4}{|c|}{ N/L } \\
\hline$<2.6$ & 79 & 28 & \multirow{2}{*}{0.06} \\
\hline$>2.6$ & 64 & 41 & \\
\hline \multicolumn{4}{|c|}{ N/P } \\
\hline$<0.02$ & 108 & 45 & \multirow{2}{*}{0.16} \\
\hline$>0.02$ & 35 & 24 & \\
\hline
\end{tabular}

Table 1: Correlation between preoperative serum CEA Level and clinicopathologic variables; CEA cut off value used is $5 \mathrm{ng} / \mathrm{ml}$ (N/L: Neutrophils to Lymphocytes ratio; N/P: neutrophils to platelets ratio).

\begin{tabular}{|c|c|c|c|}
\hline Variables & Low ca19.9 & High ca19.9 & $\mathbf{P}$ \\
\hline \multicolumn{4}{|c|}{ Age } \\
\hline$<65$ & 9 & 7 & \multirow{2}{*}{0.8} \\
\hline$>65$ & 34 & 28 & \\
\hline \multicolumn{4}{|c|}{ Gender } \\
\hline Female & 16 & 17 & \multirow{2}{*}{0.4} \\
\hline Male & 27 & 18 & \\
\hline \multicolumn{4}{|c|}{ Tumour Site } \\
\hline Right & 16 & 16 & \multirow{4}{*}{0.53} \\
\hline Left & 16 & 11 & \\
\hline Rectum & 9 & 8 & \\
\hline Transverse & 2 & 0 & \\
\hline \multicolumn{4}{|c|}{ T stage } \\
\hline 1 & 4 & 2 & \multirow{4}{*}{0.3} \\
\hline 2 & 6 & 3 & \\
\hline 3 & 30 & 23 & \\
\hline 4 & 3 & 7 & \\
\hline \multicolumn{4}{|c|}{ N stage } \\
\hline 0 & 23 & 16 & \multirow{4}{*}{0.65} \\
\hline 1 & 12 & 10 & \\
\hline 2 & 8 & 8 & \\
\hline 3 & 0 & 1 & \\
\hline \multicolumn{4}{|c|}{ N stage } \\
\hline Pos & 20 & 19 & \multirow{2}{*}{0.6} \\
\hline Neg & 23 & 16 & \\
\hline \multicolumn{4}{|c|}{ Lymph. Harvasted } \\
\hline$<12$ & 11 & 11 & \multirow{2}{*}{0.7} \\
\hline$>12$ & 32 & 24 & \\
\hline \multicolumn{4}{|c|}{ Sieric Albumin } \\
\hline$<3.5$ & 11 & 11 & \multirow{2}{*}{0.65} \\
\hline$>3.5$ & 30 & 21 & \\
\hline \multicolumn{4}{|c|}{ N/L } \\
\hline$<2.6$ & 24 & 19 & \multirow{2}{*}{0.8} \\
\hline$>2.6$ & 19 & 15 & \\
\hline \multicolumn{4}{|c|}{ N/P } \\
\hline$<0.02$ & 26 & 26 & \multirow{2}{*}{0.2} \\
\hline$>0.02$ & 17 & 8 & \\
\hline
\end{tabular}

Table 2: Correlation between preoperative serum CA19.9 Level and clinicopathologic variables; Ca 19.9 cutoff value used is $35 \mathrm{Ui} / \mathrm{ml}$ (N/L: Neutrophils to Lymphocytes ratio; N/P: neutrophils to platelets ratio).

\section{Statistical analyses}

Statistical analyses were conducted using PRISM software and Med Calc Software. Differences between groups were tested with a Chi-square test or T student. Survival rates were calculated using the Kaplan-Meier method, and prognostic factors and survival curves were compared using the log-rank test. Variables, with a statistical $\mathrm{P}$-value less than 0.05 in the univariate analysis, were entered into a multivariate Cox regression analysis. A P-value $<0.05$ was deemed to be statistically significant. Roc curves were also performed with the statistical software.

\section{Results}

Tumor location was 94 times on ascending colon (42\%), 13 on transverse colon (5.8\%), 77 on descending colon (34.4\%) and 40 on rectum (17.8\%). All tumors were histologically diagnosed as adenocarcinoma of the colon-rectum and staged according to the TNM staging system. Among the 224 investigated patients, there were 12 patients (5.3\%) who were double positive for CEA and CA19-9, 58 patients (25.9\%) who were positive for CEA only, and 23 patients (10.3\%) who were positive for CA 19-9 only. Preoperative serum CEA level was significantly associated only with $\mathrm{T}$ stage and serum albumin level; whereas there were not statistically significant differences between preoperative serum CA19.9 and patients' clinical pathologic characteristics as shown in Tables 1 and 2. 
Citation: Graziosi L, Elisabetta M, Rebonato A, Donini A (2018) Preoperative Serum Markers Prognostic Evaluation in Colon Cancer Patients. J Cancer Sci Ther 10: 022-026. doi:10.4172/1948-5956.1000511

\section{Roc curves analysis}

CEA sensitivity and specificity for 2 years OS were respectively $62.5 \%$ and $85.7 \%$ as shown in Figure 1; whereas Figure 2 showed CEA sensitivity and specificity for 1-year DFS, which is respectively 50 and 93\%. Ca19.9 Roc curves were made as well as for CEA, and they are shown in Figures 3 and 4 with a 2 -Y OS sensitivity and specificity respectively of 71.4 and $81.2 \%$, and 1-Y DFS was respectively of 64.7 and $71.7 \%$.

\section{Survival analysis}

5 Y-OS and 5Y-DFS of our series were respectively of $60 \%$ and $75 \%$. $5 Y-O S$ of patients with a low preoperative CEA level was $70 \%$ vs. $45 \%$ of patients with high CEA level. The difference was statistically different with a $\mathrm{p}<0.05$ (Figure 5). Also, DSF showed (Figure 6) a statistically significant difference with a percentage of $90 \%$ vs $65 \%$ in low and high groups respectively. Overall survival sub analysis showed also statistically significant differences $(\mathrm{p}<0.05)$ in T3-T4, N0, N+ patients according to CEA values (Figures 7a-7c). No statistically significant difference was shown in T1-2 patients according to CEA value. Similar analyses

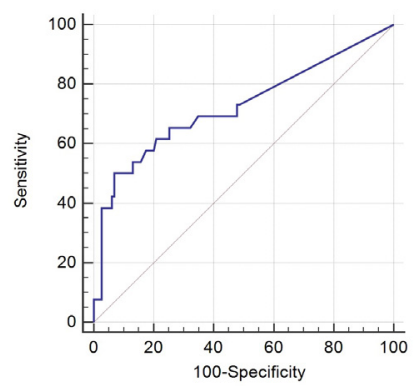

Figure 2: CEA sieric value (cutoff $5 \mathrm{ng} / \mathrm{ml}$ ) and 1 Y-DSF Roc CURVE; Sensitivity $50 \%$, Specificity $93 \%$.

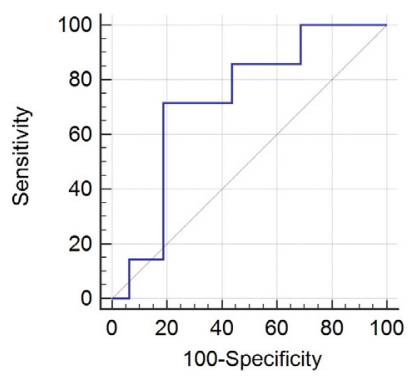

Figure 3: CA19.9 sieric value (cutoff $35 \mathrm{Ul} / \mathrm{ml}$ ) and 2Y-OS Roc CURVE; Sensitivity $71.4 \%$, Specificity

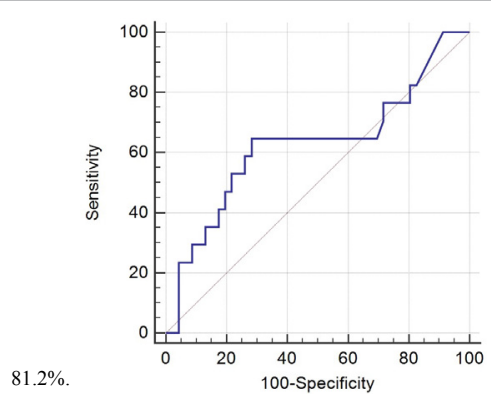

Figure 4: CA 19.9 sieric value (cutoff $35 \mathrm{UI} / \mathrm{ml}$ ) and 1Y-DSF Roc CURVE; Sensitivity $64.7 \%$, Specificity $71.7 \%$.

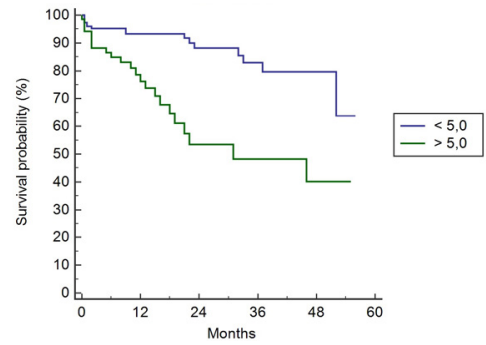

Figure 5: 5 Y-OS according to Sieric CEA Level $70 \%$ vs $45 \%$ respectively in patietns dichotomized according to the normal sieric CEA value $(5 \mathrm{ng} / \mathrm{ml})$.

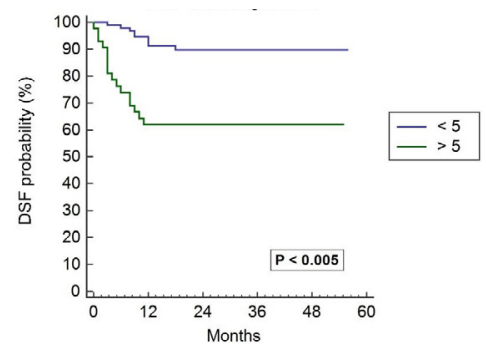

Figure 6: DSF according to Sieric CEA Level $90 \%$ vs $65 \%$ respectively in patietns dichotomized according to the normal sieric CEA value $(5 \mathrm{ng} / \mathrm{ml})$.
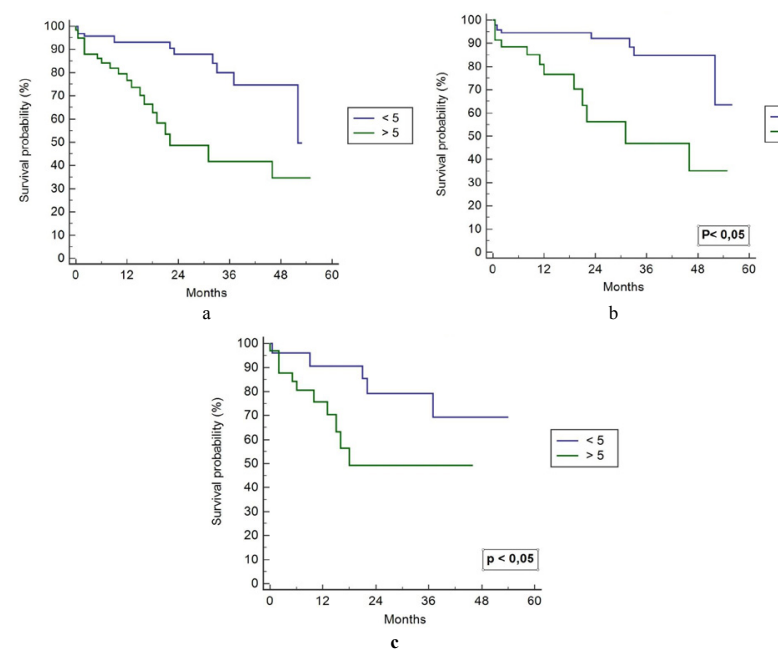

Figure 7: Overall survival in patients dichotomized according to the normal sieric CEA value (5 ng/ml); A) T3-4 patients; B) N0 patients C) $\mathrm{N}+$ patients.

were done according to preoperative CA 19.9 serum level. Figure 8 shows a 5 Y-OS of $65 \%$ vs. $35 \%$ respectively in patients with normal or altered Ca19.9 with a statistically significant difference $(\mathrm{p}<0.05)$. Regarding this marker there were statistically significant differences again in T3-4 patients, but not in T1 and T2 ONES. On the contrary, there was a statistically significant difference in N0 patients, but not in N+ (Figures 9a and 9b). Finally, we compared the overall survival of double positive patients (CEA+, CA 19-9+) with single positive, and we found a statistically significant difference $(\mathrm{P}<0.05)$. The double positive had a much worse prognosis than the single positive (Figure 10). At the multivariate analysis, both the preoperative CEA and Ca19.9 resulted as independent prognostic factors for survival with a $\mathrm{p}<0.05$.

\section{Discussion}

Tumor markers are substances that are produced by tumor cells 
and after secreted into the circulation in detectable amounts. Most of them are greatly limited for screening the asymptomatic population, being neither sensitive enough nor specific enough to detect early disease and small tumors [6]. CEA and Ca 19.9 are the most common and popular neoplastic markers investigated in colorectal patients and they are used routinely in the management of this disease. CEA is a high-molecular weight glycoprotein among the immunoglobulin superfamily of molecules that plays a pivotal role in cellular biological phenomena as adhesion, immunity or apoptosis of the tumor cells [7].

Ca 19.9 exerts its function as an adhesion molecule playing an important role in the process of the progression and invasion of the tumor. In spite of previous investigations, the survival significance of these neoplastic markers remains controversial, and it remains unclear whether monitoring them brings any clinical benefit in the management of colorectal cancer patients [8]. Pre-operative CEA is incorporated into many useful prognostic scoring systems to stratify patients at high risk of recurrences, localized in the liver after curative surgery. On the other hand, Ca 19.9 shows lesser prognostic value, if evaluated alone; for this reason, itis not so frequently used in the management of colorectal cancer patients. It could be useful to evaluate it in combination with CEA for monitoring recurrences. However, the American Society of Clinical Oncology suggests that there are too few clinical studies and evidence for using Ca 19.9 in monitoring colorectal patients. In our study we would like to investigate the prognostic value of survival and disease-free survival of pre-operative serum CEA and Ca19.9 correlating them to clinical and pathological patients' features.

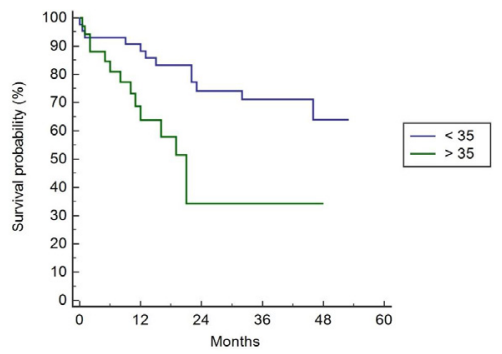

Figure 8: 5 Y-OS according to preoperative sieric CA19.9 Level $65 \%$ vs $35 \%$ respectively in patients dichotomized according to the normal sieric CA19.9 value $(35 \mathrm{Ul} / \mathrm{ml})$

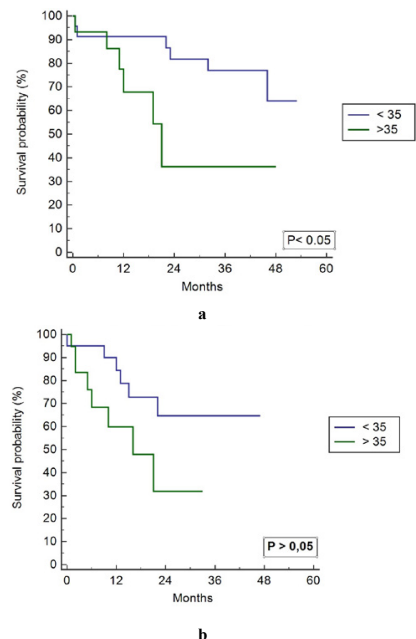

Figure 9: Overall survival in patients dichotomized according to the normal sieric Ca 19.9 value $(35 \mathrm{Ul} / \mathrm{ml})$ : a) N0 patients b) $\mathrm{N}+$ patients.

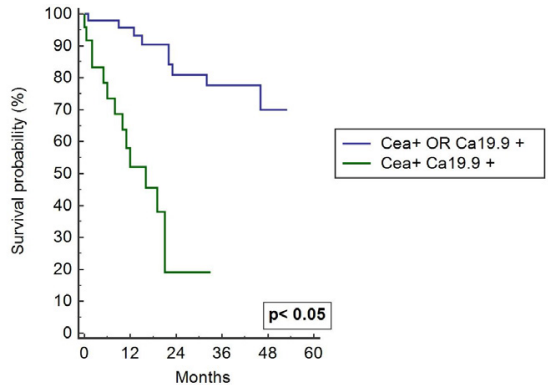

Figure 10: Overall survival in patients with double positive preoperative markers.

Actually, it is well accepted that they are not specific for the detection of colon cancer and they cannot be monitored for the detection of an adenocarcinoma in situ. On the other hand, CEA is often elevated in advanced colon disease mainly in the metastatic one. This fact is well illustrated in the work of Levy et al. [9], in which he stated that CEA and Ca 19-9 are statistically and significantly different in early and metastatic colorectal cancer, where CEA plays a role in the metastatic process. The role of CEA in cancer dissemination was revealed in a study by Hostetter et al. [10], who showed that the rate of liver metastasis in mice transplanted with colorectal tumours strongly increased after CEA injection in the murine model. Several studies showed that more CEA per gram of total protein was produced by well-differentiated colorectal cancers than by poorly differentiated specimens [11-13]. Serum CEA has also been reported to trend higher in patients with welldifferentiated tumors than in those with poorly differentiated tumors [14]. Not all the advanced colon cancers show an elevation of CEA due to the biological tumoral characteristics. This fact was confirmed by the review written by Duffy [15] who suggested that a lack of differentiation or poor differentiation might explain why some patients, with advanced colorectal cancer, didn't show increased serum concentrations of CEA. On the other hand, our results suggested that CEA elevation is not statistically correlated to tumor differentiation or histological type ( $p>0.05)$. Nakatani [16] and his colleagues demonstrated that the cancer located at the sigma showed higher concentrations of CEA and $\mathrm{Ca}$ 19.9 compared to the other cancer side. In our series both CEA and Ca 19.9 are not correlated in a statistically significant way to cancer side ( $\mathrm{p}>0.05$ ). Basing on 1y-DFS- ROC curve, pre-operative elevated CEA shows a strong importance to predict recurrences at one year after curative surgery (CEA specificity: $93 \%$ ). Thus, it allows to stratify patients before the surgical procedure, discriminating patients at high risk of recurrences who could benefit of a chemotherapy strategy or a stricter follow-up after radical surgery. Previous reports showed a significant association between elevated serum $\mathrm{Ca}$ 19-9 and poor prognosis related to disease stage in the preoperative setting. The association of $\mathrm{Ca} 19-9$ with prognosis was found to be better than the CEA [17]. In contrast, other publications have reported that the use of Ca 19-9 was very limited. The antigen was found to have no value in the screening of colon cancer patients because its positive predictive value was less than $1 \%$. [18]. In our study, Ca 19-9 did not show a big sensitivity and specificity both for OS and DFS.

In our studied patients, a pre-operative elevation of serum Ca 19.9 could be important for N0 patients who regularly are not addressed to chemotherapy. An elevation of Ca 19.9 could represent a signal to guide in these patients a more aggressive medical therapy or a more aggressive post-surgical follow-up. Our study has some limitations because, first, it includes a small number of patients; secondly it is a retrospective work, and thirdly a long-term follow up is required to provide more prognostic information. 
Citation: Graziosi L, Elisabetta M, Rebonato A, Donini A (2018) Preoperative Serum Markers Prognostic Evaluation in Colon Cancer Patients. J Cancer Sci Ther 10: 022-026. doi:10.4172/1948-5956.1000511

\section{Conclusion}

Given that colorectal cancer is a common cause of death worldwide, an effort either to achieve early diagnosis or to identify patients with poor prognosis in the preoperative period is needed to support patient management after surgical treatment. Tumoral markers are known not to be suitable in population screening for their limitations, and our results confirmed the low sensitivity of both CEA and Ca 19.9. In the present study, we found that preoperative serum CEA might advice an appropriate post-surgical follow-up when the patient is at high risk of recurrences. Whereas pre-operative Ca19.9 might indicate a very poor prognosis in N0 patients, suggesting a potential benefit of chemotherapy in these patients. We therefore recommend routine preoperative tests to evaluate both serum CEA and Ca19.9 in colorectal cancer.

Further studies into the molecular basis of tumor biology might contribute the current understanding of the nature of these tumor markers.

\section{Article Highlights}

\section{Research background}

Being the second worldwide tumor related cause of death in women and the third in men, colon cancer remains a main issue of the health care system.

\section{Research motivation}

Although pathologic stage is useful and essential for predicting prognosis in CRC patients, it is difficult to accurately determine the stage prior to the surgical treatment.

\section{Research objectives}

To identify promising prognostic factors that could preoperatively identify patients at a high risk of recurrences after surgery or with a bad survival prognosis.

\section{Research methods}

We retrospectively analyzed data on two hundred and thirtyone patients with colorectal cancer who underwent macroscopically curative surgery. Statistical analyses were conducted using PRISM software and Med Calc Software. Differences between groups were tested with a Chi-square test or T student. Survival rates were calculated using the Kaplan-Meier method, and prognostic factors and survival curves were compared using the log-rank test. A P-value $<0.05$ was deemed to be statistically significant.

\section{Research results}

Preoperative serum CEA might suggest that when the patient is at high risk of recurrences, he needs an appropriate post-surgical follow-up. Whereas the pre-operative Ca19.9 might indicate a very poor prognosis in N0 patients, suggesting a potential benefit of chemotherapy in these patients

\section{Research conclusion}

We therefore recommend routine preoperative tests to evaluate both serum CEA and Ca19.9 in colorectal cancer.

\section{Research perspectives}

Further studies into the molecular basis of tumor biology might contribute the current understanding of the nature of these tumor markers.

\section{References}

1. Ferlay J, Steliarova-Foucher E, Lortet-Tieulent J, Rosso S, Coebergh JW, et al. (2013) Cancer incidence and mortality patterns in Europe: estimates for 40 countries in 2012. Eur J Cancer 49: 1374-1403.

2. Leon P, lovino MG, Giudici F, Sciuto A, de Manzini N, et al. (2018) Oncologic outcomes following laparoscopic colon cancer resection for T4 lesions: A casecontrol analysis of 7-years' experience. Surg Endosc 32: 1133-1140.

3. Takagawa R, Fujii S, Ohta M, Nagano Y, Kunisaki C, et al. (2008) Preoperative serum carcinoembryonic antigen level as a predictive factor of recurrence after curative resection of colorectal cancer. Ann Surg Oncol 15: 3433-3439.

4. Wood CB, Ratcliffe JG, Burt RW, Malcolm AJ, Blumgart LH, et al. (1980) The clinical significance of the pattern of elevated serum carcinoembryonic antigen (CEA) levels in recurrent colorectal cancer. Br J Surg 67: 46-48.

5. Chau I, Allen MJ, Cunningham D, Norman AR, Brown G, et al. (2004) The value of routine serum carcino-embryonic antigen measurement and computed tomography in the surveillance of patients after adjuvant chemotherapy for colorectal cancer. J Clin Oncol 22: 1420-1429.

6. Aziz KJ, Maxim PE (1993) The FDA's perspective on the evaluation of tumor marker tests. Clin Chem 39: 2439-2443.

7. Hammarstrom S (1999) The carcinoembryonic antigen (CEA) family: Structures suggested functions and expression in normal and malignant tissue. Semin Cancer Biol 9: 67-81.

8. Morita S, Nomura T, Fukushima Y, Morimoto T, Hiraoka N, et al. (2004) Does serum ca 19-9 play a practical role in the management of patients with colorectal cancer? Dis Colon Rectum 47: 227-232.

9. Levy M, Visokai V, Lipska L, Topolcan O (2008) Tumor markers in staging and prognosis of colorectal carcinoma. Neoplasma 55: 138-142.

10. Richard B, Hostetter Lazel B, Ramy A, Kefung M, Fan CD, et al. (1990) Carcinoembryonic antigen as a selective enhancer of colorectal cancer metastasis. J Natl Cancer Inst 82: 380-385.

11. Polat E, Duman U, Duman M (2014) Diagnostic value of preoperative serum carcinoembryonic antigen and carbohydrate antigen 19-9 in colorectal cancer. Curr Oncol 21: 1-7.

12. Bhatnagar J, Tewari HB, Bhatnagar M, Austin GE (1999) Comparison of carcinoembryonic antigen in tissue and serum with grade and stage of colon cancer. Anticancer Res 19: 2181-2187.

13. Rieger A, Wahren B (1975) cea levels at recurrence and metastases; importance for detecting secondary disease. Scand J Gastroenterol 10: 869-874.

14. Goslin R, O'Brien MJ, Steele G (1981) Correlation of plasma cea and cea tissue staining in poorly differentiated colorectal cancer. Am J Med 71: 246-253.

15. Duffy MJ (2001) Carcinoembryonic antigen as a marker for colorectal cancer: Is it clinically useful? Clin Chem 47: 624-630.

16. Nakatani $H$ (2012) High serum level of both carcinoembryonic antigen and carbohydrate antigen 19.9 in a patient with sigmoid colon cancer without metastases. J Med Investigation 59

17. Filella X, Molina R, Grau JJ (1992) Prognostic value of ca 19.9 levels in colorectal cancer. Ann Surg 216: 55-59.

18. Steinberg W (1990) The clinical utility of the ca 19-9 tumor associated antigen Am J Gastroenterol 85: 350-355. 